\title{
MS106.P06
}

\section{Crystallographic study of human tooth enamel crystal defects}

José Reyes-Gasga ${ }^{1}$, Etienne Brès ${ }^{2}$, Joseph $^{3}$

${ }^{1}$ Instituto De Física, UNAM, Mexico City, Mexico, ${ }^{2}$ UMET, Bâtiment C6. Université de Lille 1, Lille, France, ${ }^{3}$ INSERM UMR_S 1121 , Faculté de Chirurgie Dentaire, Université de Strasbourg, Strasbourg, France

E-mail: jreyes@fisica.unam.mx

Human tooth enamel (HTE) is composed of 96 wt\% of an inorganic phase [1] consisting of crystalline carbonated hydroxyapatite crystals with an elongated prismatic shape (Figure 1). Hydroxyapatite (HAP) is the inorganic mineral, with composition $\mathrm{Ca}(\mathrm{I}) 4 \mathrm{Ca}(\mathrm{II}) 6(\mathrm{PO} 4) 6(\mathrm{OH}) 2$ and with space group $\mathrm{P} 63$. The $\mathrm{OH}$ - ions are located on the 63 axis parallel to the $\mathrm{C}$ axis and surrounded by two equilateral $\mathrm{Ca}$ (II) atom triangles directed in opposite directions, all enclosed by six $\mathrm{Ca}(\mathrm{I})$ atoms. HAP in HTE forms elongated-plate-like crystals of $30 \mathrm{~nm}$ to $60 \mathrm{~nm}$ wide and $100 \mathrm{~nm}$ to $300 \mathrm{~nm}$ long, approximately [2]. These crystals are organized in microns-in-size structures named "prism", that run from the enamel-dentin junction to the tooth enamel surface.

During the carious dissolution process, HTE crystals are first attacked on their basal planes leading to the formation of initial hexagonal holes aligned at equal distance along the [11-20] direction [3]. After, the holes join to form a single hexagonal hole elongated along the [11-20] direction while the hole develops anisotropically along the [0001] direction [3]. Up to this stage, the demineralisation process is reversible and the holes can be refilled through a remineralisation mechanism. Beyond this stage, the demineralisation process is irreversible: the central hole opens and the crystals break open.

The structural defect of nanometric width mentioned above is registered in the central region of the HTE crystallites when they are observed by HRTEM and, then, it is named "central dark line" (CDL). The CDL is presented as a contrast line always parallel to the $\left(\begin{array}{llll}1 & 0 & -1 & 0\end{array}\right)$ planes. Because these planes are observed both along the [0001] and [ $\left.\begin{array}{llllll}1 & -2 & 0\end{array}\right]$ directions, the $\mathrm{CDL}$ is in fact a plane. The authors are convinced that the knowledge of the CDL structure, properties and chemical composition is crucial for understanding of the carious process.

Crystallographic study at angstrom resolution is done on images of the human-tooth-enamel (HTE) crystallites obtained using aberration-corrected high resolution transmission electron microscopy (HRTEM) and atomic resolution scanning transmission electron microscopy (AR-STEM) in the modes of bright field (BF), annular dark field (ADF) and high angle annular dark field (HAADF). The images indicate that the central dark line (CDL) defect observed at around the center of the HTE crystals has a thickness of $0.2 \mathrm{~nm}$, approximately, and goes through one of the OH hydroxyapatite ions.

We thank Prof. Gustaaf Van Tendeloo for ESTEEM support at EMAT, University of Antwerpen (Belgium), and Prof. Johan Verbeeck for comments and suggestions. We also thank Dr. Stuart Torner and Dr. Yinggang Lu for the technical support and so practical suggestions. This research has received funding from the European Union Seventh Framework Programme under Grant Agreement 312483-ESTEEM2 (Integrated Infrastructure Initiative-I3). We also thank Samuel Tehuacanero Nuñez for TEM analysis. We thank to DGAPA-UNAM (contract IN109516) for economical support.

1. LeGeros, R. Z. (1991). Calcium phosphates in oral biology and medicine, S. Karger, AG. Basel.

2. Reyes-Gasga, J. et al. (2013) Materials Science and Engineering C 33, 4568-4574.

3. H. Tohda, H. et al. (1987). J. Dent Res. 66, 1647-1653.

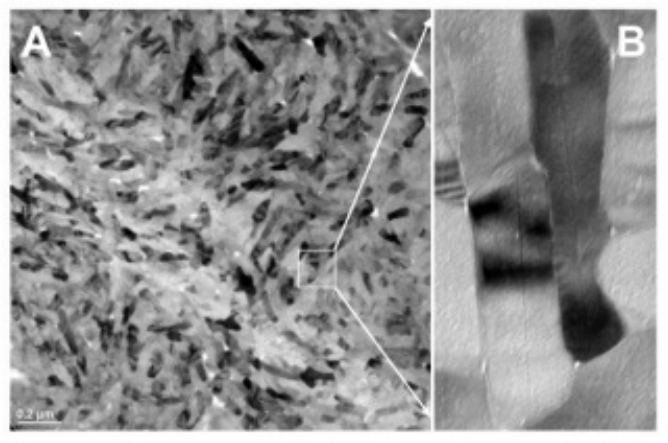

Figure 1: Dimensions and shape of the HTE crystals. A) TEM image of human tooth enamel crystals inside the micron-sized "prism". B) Magnification of the human tooth enamel in the inset. Note the presence of the CDL.

Keywords: Human tooth enamel crystals, Central dark line defect, Cs-corrected HRTEM. 